\title{
Anisotropic blind image quality assessment: Survey and analysis with current methods
}

\author{
Salvador Gabarda ${ }^{\mathrm{a}}$, Gabriel Cristóbal ${ }^{\mathrm{a}}$, Navdeep Goel ${ }^{\mathrm{b}}$ \\ ${ }^{a}$ Instituto de Óptica Daza de Valdés (CSIC), Serrano 121, 28006 Madrid, Spain \\ ${ }^{b}$ ECE Section, Yadavindra College of Engineering, Talwandi Sabo-151302, Punjab, India
}

\begin{abstract}
The Anisotropic Quality Index (AQI) was previously introduced by the authors as a non-distortion-specific, noreference image quality measure. AQI is based on measuring the variance of the expected entropy of a given image over a predefined set of orientations. In this paper, a statistical evaluation of AQI is provided using standard benchmarking parameters showing good match with observer's response. Some AQI extensions are proposed for tackling ill-posed noise types that provide fair or poor performance. A comparison with other no-reference methods has been included showing good competitiveness with many of them.
\end{abstract}

Keywords: Image quality assessment, Pseudo-Wigner distribution, Rényi entropy, anisotropy, correlation, Mean opinion scores

\section{Introduction}

No-reference image quality assessment (NR-IQA) methods provide quality estimates without any prior knowledge about the reference image and the quality evaluation is only based on the test images. Most of the conventional NR-IQA algorithms can be classified into three different categories: (i) Distortion-specific (Single/Multiple distortion), (ii) Natural scene statistics (NSS), and (iii) Training/learning methods. For recent surveys of no-reference image quality assessment algorithms, the readers may refer to [1]. This paper is focused on assessing the performance of the distortionspecific (multiple distortion mode), no-reference index called Anisotropic Quality Index (AQI) introduced by he authors in [2]. The major contributions of this paper are twofold. First, the AQI index has been evaluated using standard benchmarking parameters not previously considered in [2]. Secondly, AQI modifications are proposed to extend the suitability of the index to more noise types.

Interestingly, the AQI measure has been used in different real-world scenarios. Sidiropoulos and Muller [3] used AQI for the quality assessment of planetary degraded images and Bos and Roggermann [4] for speckle image reconstruction. In [5], AQI was applied in different biometric applications (face, fingerprints and hand veins). AQI was used in different surveillance scenarios under the presence of atmospheric distortions [6] and for biomedical image classification [7].

In a recent work, Maddheshiya [8] pointed out the need to develop no-reference quality metrics such as AQI that can overcome the limitation of other measures that are distortion specific. In addition to that, the AQI performance was validated using the LIVE database. LIVE is a standard image database that is frequently used as a benchmark because the distorted images have been evaluated by human observers. In such work, they concluded that AQI is highly correlated with Structural Similarity Index Measure (SSIM) [9] and therefore it will be suitable for no reference quality assessment and among the five distortion types included in LIVE it provides a more accurate and stable performance for white noise and Gaussian blur distortions. This known AQI limitations led us to introduce few extensions for improving the index performance.

This paper is structured as follows. Section 2 describes the AQI comparison against Differential Mean Opinion Scores (DMOS) for different distortion types of the LIVE (Laboratory for Image \& Video Engineering - University of Texas at Austin) database. Section 3 presents a statistical evaluation of the AQI performance vs Mean Opinion Scores (MOS), for the set of noise types in the Tampere Image Database 2013 (TID2013) introducing some AQI modifications to fit the measure with ill-posed types. A comparison of scoring tests with 
other existing no-reference quality assessment methods is presented in Section 4. An evaluation with the LIVE 3D database is presented in Section 5. Finally, conclusions are drawn in Section 6.

\section{The Anisotropic Quality Index and the LIVE im- age database}

\subsection{Automatic image quality assessment}

Image quality approaches are largely dependent on the area of imaging considered. However it would be of interest to define a generic objective quality assessment based on a physical measure such as the image anisotropy which relies on the assumption that high quality images have richer information contents than low quality images [3]. Additionally, it was experimentally shown that such physical parameter correlates well with the human observer's scores specially in the case of Gaussian noise and blur [2]. Based on such principle, the AQI was initially proposed in [2] as an image quality objective measure extracted from the spatial/spatialfrequency domain. In this paper, few adaptive AQI extensions are introduced by the authors for tackling illposed image distortions for improving the quality measure performance.

\subsection{The original AQI algorithm}

The AQI measure is based on the extraction of the Rényi entropy from a joint spatial/spatial-frequency representation, specifically from the Wigner Distribution (WD) [10-12]. The Rényi entropy is given by

$$
R_{\alpha}(X)=\frac{1}{1-\alpha} \log _{2}\left(\sum_{i=1}^{I} P^{\alpha}\left(x_{i}\right)\right)
$$

where $\alpha$ is a real-positive number. The Rényi entropy will be calculated by means of Eq. (1) through a Wigner Distribution:

$$
W(n, k)=2 \sum_{m=-\frac{N}{2}}^{\frac{N}{2}-1} z(n+m) z^{*}(n-m) e^{-i 2 \pi k\left(\frac{2 m}{N}\right)}
$$

In Eq. (2), $z(n)$ represents the gray values of pixel $n$ in a given image $z$. Variables $n$ and $k$ represent the spatial and spatial-frequency discrete variables respectively, and $m$ is a shifting parameter, which is also discrete. The general case of the Rényi entropy given by Eq. (1), is particularized by identifying $P$ with a normalized WD [2] to yield

$$
R(n)=-\frac{1}{2} \log _{2}\left(\sum_{k} \widetilde{W}^{3}(n, k)\right)
$$

Here $\alpha=3$ has been selected for reducing the interference of cross-terms and the normalized WD is given by

$$
\widetilde{W}(n, k)=\frac{W^{2}(n, k)}{\sum_{k} W^{2}(n, k)}
$$

For each pixel, an oriented 1-D window was used to measure the entropy along different orientations. The algorithm has a directional dependence. In a continuous support the number of possible orientations is infinite. However, in a local digital context with $\mathrm{N}$ aligned pixels, the possible orientations over the image are practically limited to a few ones, e.g., orientation between 30 and 60 degrees may incur into a great amount of overlapping pixels on an small window. Here AQI was calculated upon six equally spaced orientations $\theta=0^{\circ}, 30^{\circ}$, $60^{\circ}, 90^{\circ}, 120^{\circ}$ and $150^{\circ}$. Such set of directions has been experimentally considered a good choice for an 8-pixel window. The variance of expected directional entropy of the image has been defined as the AQI measure,

$$
A Q I \equiv \sigma(\bar{R}(\theta))
$$

where $\bar{R}(\theta)$ represents the local pixel-wise directional entropy for the whole image (see [2] for details).

\subsection{Adaptive AQI algorithm}

However, AQI offers different performance upon the type of distortion, which motivates the introduction of the modifications shown in this paper for extending the number of types of distortions able to be measured by this method.

In this paper, AQI is tested vs Differential Mean Opinion Score (DMOS) for LIVE database and the results obtained for different distortions types included in the LIVE image database are shown in Table 1. Correlation measures for JPEG have been improved with respect to the original AQI by the use of a weighting factor, as it was initially described in [2]. In practice, let us suppose that $L$ is the size of the image and $S$ is the number of directions used to measure the image's anisotropy. Let us suppose that $K$ is the total number of zero-entropy values found in the $S$ orientations while measuring the entropy. The proposed corrected measure is defined as

$$
A Q I_{J P E G}=A Q I \times\left(1-\left(\frac{K}{S L}\right)^{\beta}\right)
$$

and experimentally it showed a better correlation with DMOS for JPEG images than the original AQI. In Eq. (6) parameter $\beta$ has been empirically determined to be 
0.1. The AQI-weigthed measure applied to JPEG compressed images provides now high correlation values in accordance with observer's response. Correlation to MOS has been achieved by means of the Spearman rank order correlation coefficient (SROCC), Pearson rank order correlation coefficient (PROCC) and Kendall rank order correlation coefficient (KROCC).

\section{The Anisotropic Quality Index and TID2013 im- age database}

\subsection{The Noise group in TID2013}

The TID2013 image database includes a list of eleven types of noise under the Noise category. Table 2 summarizes the AQI performance for such noise types, using the original definition given by Eq. (5) and their correlation values have been computed using the Mean Opinion Score (MOS) provided by the observer's score [13].

Table 2 reveals that the AQI is a suitable noreference measure for most of the noise types included in TID2013 with exception of three cases: (i) 3. Spatially correlated noise, (ii) 5. High frequency noise and (iii) 7. Quantization noise. For these noises, enough correlation has not been achieved and therefore such kind of noise type cannot be properly evaluated by the AQI. In order to extend the AQI to the noise types not covered by the original algorithm, some modifications are needed as discussed next.

\subsection{AQI modification for spatially correlated noise and High Frequency noise}

A well-known example of spatially correlated noise is speckle noise, which is the granular or mottled phenomenon that appears, e.g., when a rough surface is illuminated by a laser source. Speckle noise violates the statistical features of original images by modifying the image moments. In order to make feasible the measurement of images affected by speckle noise, the AQI has been normalized through the expected value of the image entropy, calculated as the mean value of the entropy along a set of orientations.

Hence, the AQI normalizing procedure is given as follows:

$$
A Q I_{\text {norm }}=\frac{A Q I}{\mu(\bar{R}(\theta))}=\frac{\sigma(\bar{R}(\theta))}{\mu(\bar{R}(\theta))}
$$

Table 3 shows the correlation measures after applying the normalized index defined by Eq. 7 for the TID2013 database Noise types.
The normalized AQI measure has improved over the original for Spatially correlated noise and additionally for High frequency noise. However, such normalized measure does not provide enough accuracy for Quantization noise (SROCC: 0.76, KROCC: 0.7120, PROCC: 0.7730 ), where KROCC was used as a measure of concordance between two sets of rankings. It is worth mentioning that the normalization procedure has a selective behavior, as Quantization noise, Gaussian blur and Image denoising are now out of the scope of the normalized measure. In other words, the results presented in Table 3 indicate that the original AQI should be preferred for these other noise types. Fig. 2a-b shows examples of images degraded with spatial correlated noise and high frequency noise respectively.

In order to provide a feasible explanation of the failed results for $A Q I_{n o r m}$ with Gaussian blur and image denoising noise types, Fig. 1 shows the results of the expected values for directional entropy $R(\theta)$ for each of the 11 different noise types described in the TID2013 database. It is worth to be mentioned that while most types showed increasing values of entropy for increasing noise (represented in the horizontal axis of Fig. 1), Gaussian blur and image denoising showed decreasing values of entropy and this characteristic has to be considered as the reason for differences in the accuracy performance. Also in the case of lossy compression of noisy images a non monotonous entropy response is observed, being this the reason for the loss of accuracy when the AQI normalized measure is used, as shown in Fig. 1.

\subsection{AQI extension for Quantization noise}

Quantization noise is a distortion originated when a signal is transformed from analog to digital form. $\mathrm{Nu}-$ merically, it represents the difference between the ana$\log$ input and the digital output that is usually called Quantization error. This type of noise is characterized by its non-linearity character and its signal-dependence. An image example degraded with Quantization noise is shown in Fig. 2c. This type of noise can be considered as a limiting case to the JPEG compression. The loss of accuracy with Spatially correlated and High frequency noises has been solved by considering the normalized AQI measure as shown in section 3.2. However, Quantization noise requires a different treatment. Experimentally, it has been observed that Quantization noise has a high amount of zero values of entropy when considered at pixel level. Hence, in order to improve the correlation values MOS vs SROCC, KROCC and PROCC for this particular case, the measure of anisotropy has been corrected replacing AQI by $A Q I_{\text {norm }}$ in Eq. 6. The quality 
Table 1: AQI vs DMOS for LIVE database release 2

\begin{tabular}{lccc}
\hline Noise type & SROCC & KROCC & PROCC \\
\hline 1. JPEG2000 & 0.8732 & 0.7981 & 0.8441 \\
2. JPEG* & 0.9075 & 0.8122 & 0.9060 \\
3. White noise in RGB components & 1.0000 & 1.0000 & 0.8999 \\
4. Gaussian blur & 0.9980 & 0.9954 & 0.9865 \\
5. Transmission error in JPEG2000 & 0.8522 & 0.7747 & 0.8637 \\
\hline (*) corrected as per Eq. (6) & 0.9262 & 0.8761 & 0.9000 \\
\hline
\end{tabular}

Table 2: AQI vs MOS for the noise group of images in TID2013

\begin{tabular}{lccc}
\hline Noise type & SROCC & KROCC & PROCC \\
\hline 1. Additive Gaussian noise & 0.9840 & 0.9680 & 0.9510 \\
2. Noise in color comp. & 0.9240 & 0.8880 & 0.9390 \\
3. Spatially correlated noise* & -0.3440 & -0.3360 & -0.3578 \\
4. Masked noise & 0.8848 & 0.8375 & 0.8942 \\
5. High frequency noise* & 0.8400 & 0.8160 & 0.7911 \\
6. Impulse noise & 0.9880 & 0.9840 & 0.9407 \\
7. Quantization noise* & -0.5360 & -0.4720 & -0.6108 \\
8. Gaussian blur & 0.9960 & 0.9920 & 0.9897 \\
9. Image denoising & 0.9789 & 0.9579 & 0.9701 \\
19. Multiplicative Gaussian noise & 0.9349 & 0.8939 & 0.9053 \\
21. Lossy compression of noisy images & 0.9480 & 0.9120 & 0.9221 \\
\hline average (3, 5 and 7 excluded) & 0.9548 & 0.9292 & 0.9390 \\
\hline
\end{tabular}

Table 3: $A Q I_{\text {norm }}$ vs MOS for the noise group of images in TID2013

\begin{tabular}{lccc}
\hline Noise type & SROCC & KROCC & PROCC \\
\hline 1. Additive Gaussian noise & 0.9840 & 0.9680 & 0.9765 \\
2. Noise in color comp. & 0.9360 & 0.9040 & 0.9561 \\
3. Spatially correlated noise & 1.0000 & 1.0000 & 0.9732 \\
4. Masked noise & 0.9368 & 0.8855 & 0.9516 \\
5. High frequency noise & 0.9640 & 0.9360 & 0.9614 \\
6. Impulse noise & 0.9960 & 0.9920 & 0.9730 \\
7. Quantization noise* & 0.7600 & 0.7120 & 0.7730 \\
8. Gaussian blur* & -0.5200 & -0.5200 & -0.4457 \\
9. Image denoising* & -0.9030 & -0.8780 & -0.8896 \\
19. Multiplicative Gaussian noise & 0.9830 & 0.9740 & 0.9564 \\
21. Lossy compression of noisy images & 0.8840 & 0.8960 & 0.8462 \\
\hline average (7, 8 and 9 excluded) & 0.9604 & 0.9444 & 0.9493 \\
\hline
\end{tabular}




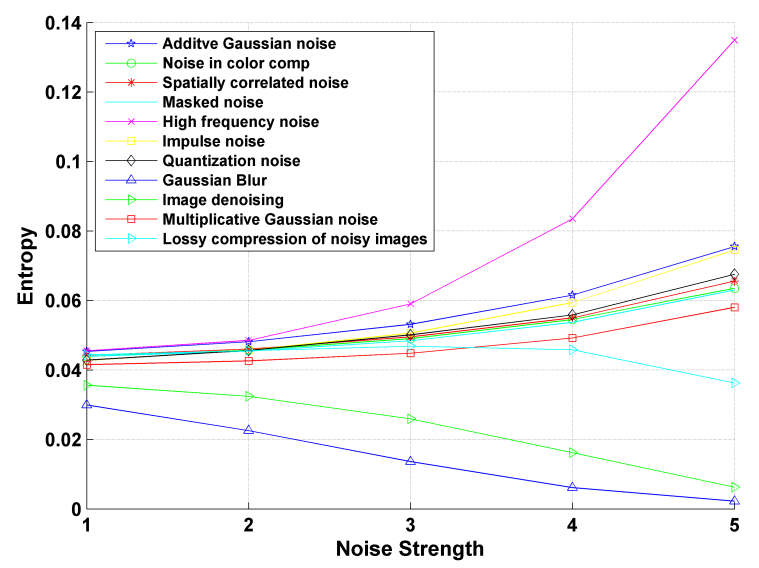

Figure 1: Entropy versus noise strength for different noise types corresponding to images from the TID2013 database

assessment measure becomes then

$$
A Q I_{\text {quant }}=A Q I_{\text {norm }} \times\left(1-\left(\frac{K}{S L}\right)^{\beta}\right)
$$

where the parameters $K, L, S$ and $\beta$ have the same meaning as in Eq. (6). Likewise, the second factor of Eq. (8) penalizes the presence of zero-entropy values in the directional entropy histograms due to the presence of flat areas in the original image after the quantization process. Parameter $\beta$ has been empirically determined to be 0.1 .

A great improvement in the correlation values has been obtained through this normalized-weighted AQI measure as shown in Table 4. The value of correlation with MOS for quantization noise obtained by the $A Q I_{\text {quant }}$ method has been compared with the Transform Domain Measure of Enhancement with Chrominance (TDMECH) [14] for the TID2008 database and has shown to provide better performance for the PROCC correlation measure (see Table 5).

\section{Comparison with other methods}

A comparison study of AQI with the most renowned NR-IQA metrics was performed including Autoregressive-based Image Sharpness Metric (ARISM) [15]; Blind Image Blur Evaluation (BIBLE) [16]; Natural Image Quality Evaluator (NIQE) [17]; Blind Image Quality Index (BIQI)[18]; Blind/Referenceless Image Spatial Quality Evaluator (BRISQUE)[19]; Distortion Identification-based Image Verity and Integrity Evaluation (DIIVINE) [20] and Spatial-Spectral Entropy-based Quality (SSEQ) [21] index methods. It should be noted that BIQI, BRISQUE,
DIIVINE and SSEQ are learning-based methods, and the images in the LIVE database are used to train a support vector regression model.

Tables 6 and 7 summarize the experimental results for non-training and training-on-LIVE measures respectively and the best results are marked in boldface. It can be observed that the AQI algorithm equals or even outperforms the other methods for Gaussian Blur and White noise and it is close to these methods in the remaining distortion types. That means that although the AQI method is not a learning based method, it is competitive when compared with other learning-based methods.

\section{The Anisotropic Quality Index and the LIVE 3D image database}

The field of NR-IQA of 2D images and videos received a lot of attention in the past decades however the development of 3D image/video quality assessment remains relatively un-explored and still in its early stage. In this section, the AQI and its corrected measure $A Q I_{J P E G}$ have been tested against the distorted $3 \mathrm{D}$ content of Phase 1 and Phase 2 dataset of the LIVE 3D image quality database [23-25] and results obtained for different distortions types are shown in Tables 8 and 9 respectively. LIVE 3D image quality database was constructed in two phases. Phase I contains only symmetrically distorted stereo images and Phase II provides both symmetrically and asymmetrically distorted stereo images annotated with subjective human rating. These results are in line with the measures obtained when testing LIVE 2D images. It is worth to note that, based on the benchmark correlation measures with DMOS scores, AQI is a good NR-IQA measure for all noise types in 
Table 4: $A Q I_{\text {quant }}$ vs MOS for quantization noise type in TID2013

\begin{tabular}{lccc}
\hline Noise Type & SROCC & KROCC & PROCC \\
\hline Quantization Noise & 0.9880 & 0.9760 & 0.9494 \\
\hline
\end{tabular}

Table 5: The comparison of $A Q I_{\text {quant }}$ and TDMECH [14] vs MOS of PROCC for quantization noise type in TID2008

\begin{tabular}{lcc}
\hline \multirow{2}{*}{ Noise Type } & \multicolumn{2}{c}{ PROCC } \\
\cline { 2 - 3 } & in $[14]$ & $A Q I_{\text {quant }}$ \\
\hline Quantization Noise & 0.7665 & $\mathbf{0 . 9 4 2 5}$ \\
\hline
\end{tabular}

Table 6: Comparison of AQI with general-purpose NR-IQA metrics for LIVE database release 2. AQIJPEG corrected as per Eq. (6)

\begin{tabular}{lcccccc}
\hline Measure & $\begin{array}{c}\text { Correlation } \\
\text { Coefficients }\end{array}$ & GB & JPEG2K & JPEG & WN & FF \\
\hline \multirow{3}{*}{ ARISM [15] } & SROCC & $\mathbf{0 . 9 9 8 0}$ & 0.9566 & $\mathbf{0 . 9 4 0 4}$ & 0.7695 & 0.7123 \\
& KROCC & $\mathbf{0 . 9 9 5 4}$ & 0.9059 & 0.8717 & 0.6919 & 0.6598 \\
& PROCC & 0.9662 & $\mathbf{0 . 9 5 6 0}$ & $\mathbf{0 . 9 3 7 3}$ & 0.8362 & 0.7490 \\
\hline \multirow{3}{*}{ BIBLE [16] } & SROCC & $\mathbf{0 . 9 9 8 0}$ & $\mathbf{0 . 9 7 9 5}$ & 0.8085 & 0.7084 & $\mathbf{0 . 9 0 1 5}$ \\
& KROCC & $\mathbf{0 . 9 9 5 4}$ & $\mathbf{0 . 9 4 5 2}$ & 0.7660 & 0.6506 & $\mathbf{0 . 8 3 4 5}$ \\
& PROCC & $\mathbf{0 . 9 8 7 4}$ & 0.8741 & 0.8080 & 0.7485 & $\mathbf{0 . 8 7 8 2}$ \\
\hline \multirow{3}{*}{ NIQE [17] } & SROCC & 0.9921 & 0.9324 & 0.8482 & 0.9961 & 0.8562 \\
& KROCC & 0.9816 & 0.8730 & 0.7213 & 0.9908 & 0.7609 \\
& PROCC & 0.9271 & 0.9179 & 0.7949 & 0.8118 & 0.8686 \\
\hline \multirow{3}{*}{ AQI } & SROCC & $\mathbf{0 . 9 9 8 0}$ & 0.8732 & $0.9262^{*}$ & $\mathbf{1 . 0 0 0 0}$ & 0.8522 \\
& KROCC & $\mathbf{0 . 9 9 5 4}$ & 0.7981 & $\mathbf{0 . 8 7 6 1}$ & $\mathbf{1 . 0 0 0 0}$ & 0.7747 \\
& PROCC & 0.9865 & 0.8441 & $0.9000^{*}$ & $\mathbf{0 . 8 9 9 9}$ & 0.8637 \\
\hline
\end{tabular}

Table 7: Comparison of AQI with general-purpose training based NR-IQA metrics for LIVE database release $2 . A Q I_{J P E G}$ corrected as per Eq. (6

\begin{tabular}{ccccccc}
\hline Measure & $\begin{array}{c}\text { Correlation } \\
\text { Coefficients }\end{array}$ & GB & JPEG2K & JPEG & WN & FF \\
\hline \multirow{3}{*}{ BIQI [18] } & SROCC & 0.9685 & 0.9315 & 0.9340 & 0.9645 & 0.8069 \\
& KROCC & 0.9356 & 0.8638 & 0.8558 & 0.9172 & 0.7012 \\
& PROCC & 0.9422 & 0.8964 & 0.8598 & 0.9283 & 0.8309 \\
\hline \multirow{3}{*}{ BRISQUE [19] } & SROCC & 0.9941 & 0.9568 & 0.9377 & $\mathbf{1 . 0 0 0 0}$ & 0.8877 \\
& KROCC & 0.9862 & 0.9165 & 0.8674 & $\mathbf{1 . 0 0 0 0}$ & 0.8161 \\
& PROCC & 0.9732 & 0.9540 & 0.8920 & 0.9704 & 0.9120 \\
\hline \multirow{3}{*}{ DIIVINE [20] } & SROCC & 0.9941 & 0.8530 & 0.8795 & 0.9842 & 0.8365 \\
& KROCC & 0.9862 & 0.7518 & 0.7704 & 0.9678 & 0.7471 \\
& PROCC & 0.9787 & 0.8529 & 0.8630 & 0.9651 & 0.8765 \\
\hline \multirow{3}{*}{ SSEQ [21] } & SROCC & 0.9961 & $\mathbf{0 . 9 7 6 6}$ & $\mathbf{0 . 9 7 3 6}$ & 0.9941 & $\mathbf{0 . 8 9 7 5}$ \\
& KROCC & 0.9908 & $\mathbf{0 . 9 4 6 1}$ & $\mathbf{0 . 9 3 1 8}$ & 0.9862 & $\mathbf{0 . 8 3 4 5}$ \\
& PROCC & $\mathbf{0 . 9 8 7 5}$ & $\mathbf{0 . 9 5 9 7}$ & $\mathbf{0 . 9 8 0 5}$ & $\mathbf{0 . 9 7 4 8}$ & $\mathbf{0 . 9 1 6 4}$ \\
\hline \multirow{3}{*}{ AQI } & SROCC & $\mathbf{0 . 9 9 8 0}$ & 0.8732 & $0.9262^{*}$ & $\mathbf{1 . 0 0 0 0}$ & 0.8522 \\
& KROCC & $\mathbf{0 . 9 9 5 4}$ & 0.7981 & $0.8761^{*}$ & $\mathbf{1 . 0 0 0 0}$ & 0.7747 \\
& PROCC & 0.9865 & 0.8441 & $0.9000^{*}$ & 0.8999 & 0.8637 \\
\hline
\end{tabular}

LIVE 2D and 3D databases, except for JPEG, for which the AQI measure has required a modification defined by $A Q I_{J P E G}$ as previously indicated.

\section{Discussion and conclusions}

A statistical evaluation of the no-reference index called Anisotropic Quality Index (AQI) introduced in [2] has been reviewed and analyzed for different degra- 
Table 8: AQI vs DMOS for LIVE 3D database: Phase I

\begin{tabular}{lccc}
\hline Noise type & SROCC & KROCC & PROCC \\
\hline 1. JPEG2000 & 0.85 & 0.7833 & 0.9251 \\
2. JPEG* & -0.0291 & 0.0361 & -0.086 \\
3. White noise in RGB components & 0.93 & 0.8833 & 0.9828 \\
4. Gaussian blur & 0.9526 & 0.9298 & 0.9283 \\
5. Transmission error in JPEG2000 & 0.83 & 0.7667 & 0.9151 \\
\hline (*) corrected measure $A Q I_{J P E G}$ & 0.6807 & 0.6337 & 0.8111 \\
\hline
\end{tabular}

Table 9: AQI vs DMOS for LIVE 3D database: Phase II

\begin{tabular}{lccc}
\hline Noise type & SROCC & KROCC & PROCC \\
\hline 1. JPEG2000 & 0.8604 & 0.75 & 0.8668 \\
2. JPEG* & -0.2808 & -0.2382 & -0.2957 \\
3. White noise in RGB components & 0.9021 & 0.7917 & 0.9089 \\
4. Gaussian blur & 0.9396 & 0.8403 & 0.9714 \\
5. Transmission error in JPEG2000 & 0.9354 & 0.8403 & 0.9289 \\
\hline (*) corrected measure AQI JPEG $^{*}$ & 0.8549 & 0.7324 & 0.8065 \\
\hline
\end{tabular}

dations and databases (LIVE and TID2013) against DMOS and MOS scores respectively. Additionally, AQI has been tested for the LIVE 3D database for different distortions. The results that are comparable with the LIVE 2D database have been summarized here. The fact that in our early experiments we obtained a significant improvement for the quality assessment of images affected by the JPEG distortion in LIVE database through a modification of the AQI measure has been the main motivation to introduce other possible modifications for adapting the AQI to different scenarios. The results of such survey have been presented and illustrated through the different sections of this communication. The AQI measure has shown a high correlation with the MOS scores in most of the distortions included in the Noise category of the TID2013 image database. Although correlation results with MOS or DMOS show special cases where AQI is not a suitable measure, it is also true that weighted or normalized versions of the original definition provide equal or even larger correlation values than other full reference metrics tested. Designing a non-distortion-specific and no-reference image quality measure is a very challenging task. The extended AQI measures can be included in the category of Reduced Reference (RR) methods when only a partial information about the types of distortions on the images are available [22]. In a real world scenario, where the distortion types cannot be determined in advance or there is a suspicion that multiple degradations are present, a good strategy will be to combine the original AQI with other quality measures as in [3]. A MATLAB toolbox for computing AQI for arbitrary grayscale or color images is available from http://bit.ly/2euSwDT. Additionaly, a Python notebook implementing the AQI algorithm is located at http://bit.ly/2Cmrc35.

\section{Acknowledgements}

This work has been partially supported by the grant CTM-2014-51907 from the Spanish Ministry of Economy and Competitivity. The authors would like to thank the anonymous reviewers for their constructive comments and suggestions. The images included in this paper have been released by the Eastman Kodak Company for unrestricted usage.

\section{References}

[1] R. Manap, L. Shao, Non-distortion-specific no-reference image quality assessment: A survey, Information Sciences 301 (2015) 141-160.

[2] S. Gabarda, G. Cristóbal, Blind image quality assessment through anisotropy, Journal of Optical Society of America A 24 (12) (2007) B42-B51.

[3] P. Sidiropoulos, J. Muller, Automatic quality assessment of planetary images, in: Proceedings of the European Planetary Science Congress, vol. 10, 2015.

[4] J. Bos, M. Roggermann, Blind image quality metrics for optimal speckle reconstruction in horizontal imaging scenarios, Optical Engineering 51 (10) (2012) 107003.

[5] M. El-Abed, C. Charrier, C. Rosenberg, Quality assessment of image-based biometric information, EURASIP J. on Image and Video Processing 3 (2015) 1-15.

[6] N. Anantrasirichai, N. Kingsbury, D. Bull, Atmospheric turbulence mitigation using complex wavelet-based fusion, IEEE Trans. on Image Processing 22 (2013) 2398-2408. 


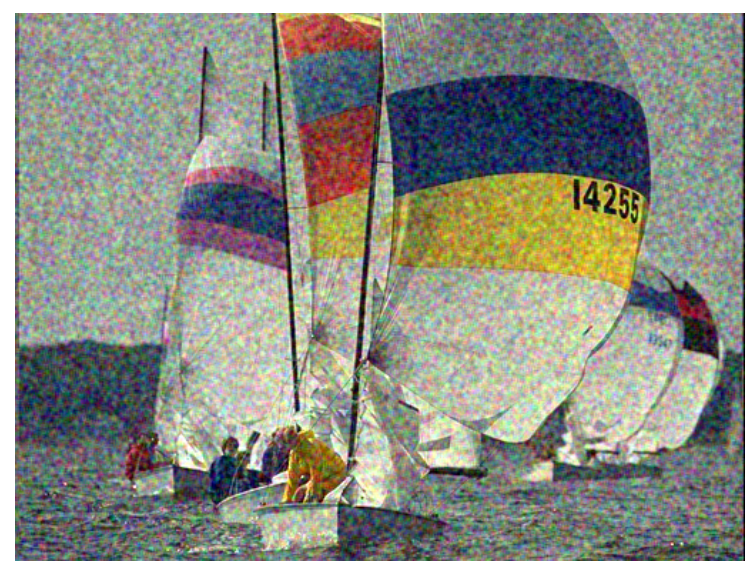

(a)

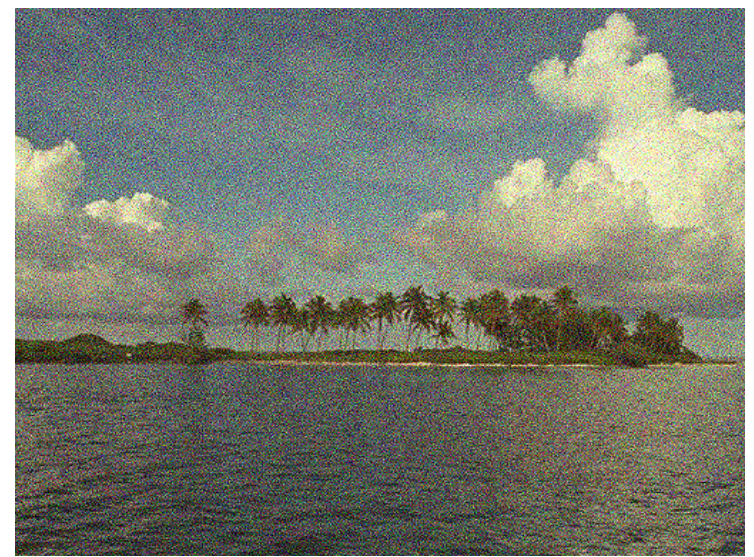

(b)

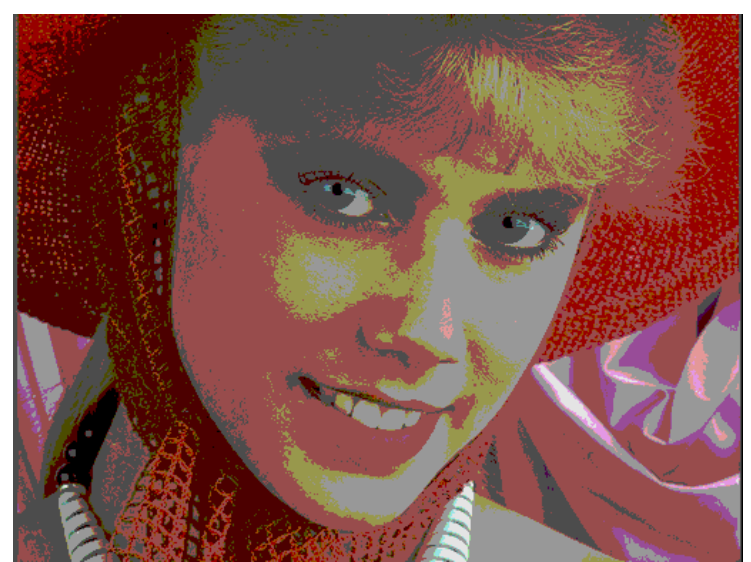

(c)

Figure 2: (a) Example of spatially correlated noise; (b) Example of a degraded image with high frequency noise; (c) Example of image degraded with quantization noise. Images have been selected from the TID2013 database.
[7] H. C. de Oliveira, B. Barufaldi, L. R. Borges, S. Gabarda, P. R. Bakic, A. D. Maidment, H. Schiabel, M. A. Vieira, Validation of no-reference image quality index for the assessment of digital mammographic images, in: SPIE Medical Imaging, International Society for Optics and Photonics, 978713-978713, 2016.

[8] A. Maddheshiya, Analysis of anisotropic blind image quality assessment, Ph.D. thesis, National Institute of Technology, Odisha, India, 2014.

[9] Z. Wang, A. C. Bovik, H. R. Sheikh, E. P. Simoncelli, Image quality assessment: from error visibility to structural similarity, IEEE Transactions on Image Processing, 4(13), 600-612, 2004.

[10] E. Wigner, On the quantum correction for thermodynamic equilibrium, Physical Review 40 (5) (1932) 749.

[11] T. Claasen, W. Mecklenbrauker, The Wigner Distribution-A Tool for Time Frequency Analysis, 1-3, Philips J. Res 35 (3) (1980) 217.

[12] K.-H. Brenner, A discrete version of the Wigner distribution function, Proc. EURASIP, Signal Processing II: Theories and Applications (1983) 307-309.

[13] N. Ponomarenko, L. Jin, O. Ieremeiev, V. Lukin, K. Egiazarian, J. Astola, B. Vozel, K. Chehdi, M. Carli, F. Battisti, et al., Image database TID2013: Peculiarities, results and perspectives, Signal Processing: Image Communication 30 (2015) 57-77.

[14] A. Samani, K. Panetta, S. Agaian, Quality assessment of color images affected by transmission error, quantization noise, and non-eccentricity pattern noise, in: IEEE International Symposium on Technologies for Homeland Security (HST), 1-6, 2015.

[15] K. Gu, G. Zhai, W. Lin, X. Yang, W. Zhang, No-reference image sharpness assessment in autoregressive parameter space, IEEE Transactions on Image Processing 24 (10) (2015) 3218-3231.

[16] L. Li, W. Lin, X. Wang, G. Yang, K. Bahrami, A. C. Kot, Noreference image blur assessment based on discrete orthogonal moments, IEEE transactions on cybernetics 46 (1) (2016) 3950.

[17] A. Mittal, R. Soundararajan, A. C. Bovik, Making a completely blind image quality analyzer, IEEE Signal Processing Letters 20 (3) (2013) 209-212.

[18] A. K. Moorthy, A. C. Bovik, A two-step framework for constructing blind image quality indices, IEEE Signal Processing Letters 17 (5) (2010) 513-516.

[19] A. Mittal, A. K. Moorthy, A. C. Bovik, No-reference image quality assessment in the spatial domain, IEEE Transactions on Image Processing 21 (12) (2012) 4695-4708.

[20] A. K. Moorthy, A. C. Bovik, Blind image quality assessment: From natural scene statistics to perceptual quality, IEEE Transactions on Image Processing 20 (12) (2011) 3350-3364.

[21] L. Liu, B. Liu, H. Huang, A. C. Bovik, No-reference image quality assessment based on spatial and spectral entropies, Signal Processing: Image Communication 29 (8) (2014) 856-863.

[22] S. Mahmoudpour and P. Schelkens, Reduced-reference image quality assessment based on internal generative mechanism using shearlets and Renyi entropy analysis, in QoMEX, Erfurt, Germany (2017) 1-5.

[23] A. K. Moorthy, C. -C. Su, A. Mittal and A. C. Bovik, Subjective evaluation of stereoscopic image quality, Signal Processing: Image Communication, 28 (8), (2013) 870-883.

[24] Ming-Jun Chen, Che-Chun Su, Do-Kyoung Kwon, Lawrence K. Cormack, Alan C. Bovik, Full-reference quality assessment of stereopairs accounting for rivalry, Signal Processing: Image Communication, 28 (9), (2013) 1143-1155.

[25] Ming-Jun Chen, Lawrence K. Cormack, Alan C. Bovik, NoReference Quality Assessment of Natural Stereopairs, IEEE Transactions on Image Processing, 28 (9), (2013) 3379-3391. 